\title{
Microglial stability and repopulation in the retina
}

\author{
T A Albini, R C Wang, B Reiser, E Zamir, G S Wu, N A Rao
}

Br J Ophthalmol 2005;89:901-903. doi: 10.1136/bjo.2004.060293

\begin{abstract}
Background/aims: Parenchymal central nervous system microglia are repopulated by bone marrow derived monocytes more slowly than any other reticuloendothelial cells. The contribution of bone marrow derived monocytes to the uninflammed retina has not been studied. The present study sought to determine repopulation of retinal microglia in uniflammed retina by bone marrow derived monocytes in bone marrow chimeric rats.

Methods: Chimeric $(\mathrm{Y} \rightarrow \mathrm{X})$ Lewis rats were constructed by transplanting $5 \times 10^{7}$ male bone marrow cells into lethally irradiated female recipient rats. The chimeras were sacrificed $8,10,12,30$, and 52 weeks after bone marrow transplant, and retina, brain, lung, and spleen samples were collected. DNA was extracted and quantified. Y positive infiltrating cells in the collected samples were detected by polymerase chain reaction amplification of a $Y$ chromosome specific $104 \mathrm{bp}$ fragment.

Results: There was a rapid repopulation of haematopoietic tissues in the spleen (at 8 weeks), confirming the establishment of chimerism, and to a lesser extent, of lung lat 30 weeks). This repopulation was absent in the brain parenchyma and retina until 52 weeks after transplantation. Conclusions: These data indicate that resident microglia in the retina, much like those in the brain, are stable in number in the retinal compartment (up to 1 year), and repopulation by bone marrow derived cells may be delayed for a year.
\end{abstract}

M icroglia are resident monocytes in the retina and central nervous system (CNS), functionally similar to macrophages. In retinal tissue, microglia can be distinguished from macrophages primarily by morphological features and perivascular distribution. These cells have long been known to have a reactive role in response to local tissue injury or degeneration. Growing evidence suggests an active proinmflammatory role for microglia as well. Resident microglia play an active proinflammatory part in the CNS in several experimental and clinical diseases, including experimental autoimmune encephalomyelitis, cerebrovascular disease, Alzheimer's disease, HIV encephalopathy, and multiple sclerosis. ${ }^{1-4}$ Although there is no direct evidence that retinal microglia are potent antigen presenting cells in vivo, retinal microglia express phenotypic markers of antigen presenting cells in vitro. ${ }^{5}$ In experimental autoimmune uveitis (EAU) microglia migrate to the photoreceptor cell layer and generate TNF $\alpha$ and peroxynitrite early in the course of disease, before macrophage infiltration. ${ }^{6}$

Monocyte precursor cells are thought to move to the CNS and the retina during embryonic life and the early postnatal period. There, these precursor cells transform into mature resting microglia of normal $\mathrm{CNS}$ and retinal tissue. ${ }^{78}$ As a consequence of inflammation or other tissue damage, bone marrow derived monocytes commonly invade the CNS or retina. In healthy CNS and retina, renewal of microglial populations may be accomplished by in situ proliferation of resident microglia. Alternatively, bone marrow derived macrophages may slowly migrate to healthy CNS or retinal tissue and transform into resident microglia. The mechanism of microglial renewal is not clearly understood in the CNS. Some CNS monocytes, primarily meningeal microglia and perivascular dendritic cells, may be slowly replaced by bone marrow derived monocytes. ${ }^{9} 10$ In contrast, parenychymal CNS microglia have been thought to be renewed predominantly by in situ proliferation of resident microglia. However, one recent study of CNS parenchymal microglia found that these cells may also be repopulated by bloodborne monocytes after peripheral nerve axotomy. ${ }^{11}$ The authors conclude that parenchymal microglia are wanderers between the peripheral immune system and the CNS. The mechanism of microglial renewal in the retina is not well described.

To test the hypothesis that retinal microglia are predominantly derived from resident microglia and not from bone marrow derived monocytes, we used a chimeric model of male Lewis rat bone marrow transplanted to lethally irradiated female Lewis rats. Immunohistochemistry or in situ hybridisation of Y chromosome gene product from serial sections may not be sufficiently sensitive to detect small numbers of bone marrow derived cells in this chimeric model. Therefore we assayed retinas, brain, lung and spleen by polymerase chain reaction (PCR) for a Y chromosome gene product at various time intervals after transplantation to determine if any bone marrow derived cells had established residence in these organs. Since monocytes would be the principal - if not only-infiltrating bone marrow derived cells in the uninflamed retina and CNS, the presence of $\mathrm{Y}$ chromosome gene product indicates infiltration of bone marrow derived monocytes into the retina. In a previous experiment, ${ }^{6}$ we found that Y chromosome gene product could not be detected in the retinas of bone marrow chimeric Lewis rats. Here, we expand these data in animals surviving for 1 year after bone marrow transplant.

\section{MATERIALS AND METHODS}

Lewis rats weighing 150 - 175 g (Charles River Laboratory, Wilmington, MA, USA) were used in all studies. Ten chimeric $(\mathrm{Y} \rightarrow \mathrm{X})$ rats were constructed according to a previously described protocol. ${ }^{6}$ Briefly, bone marrow removed from the tibia, femur, and humerus of the male donor rats was passed through a density gradient medium (Ficoll-Paque Plus; Amersham Pharmacia Biotech, Piscataway, NJ, USA) to remove red blood cells and cell debris. The cells were washed with RPMI 1640 (Gibco BRL, Rockville, MD, USA) supplemented with $10 \%$ fetal bovine serum. Female rats were irradiated with 1000 rads by a caesium-135 gamma irradiator (Gamma Cell-40; Atomic Energy of Canada, Ltd, Ottawa, Ontario, Canada) and then given intracardiac injections of $5 \times 10^{7}$ bone marrow cells in $1.0 \mathrm{ml}$ Hanks' balanced salt $^{\prime}$ solution.

Abbreviations: CNS, central nervous system; EAU, experimental autoimmune uveitis 
Table 1 Detection of Sry (Y chromosome) gene product by PCR in solid organs harvested from two bone marrow chimeric $(Y \rightarrow X)$ Lewis rats for each of five time points after bone marrow transplant

\begin{tabular}{|c|c|c|c|c|c|}
\hline & 8 weeks & 12 weeks & 15 weeks & 30 weeks & 52 weeks \\
\hline $\begin{array}{l}\text { Spleen } \\
\text { Lung } \\
\text { Right retina } \\
\text { Left retina } \\
\text { Brain }\end{array}$ & $\begin{array}{l}+1+ \\
-1- \\
-1- \\
-1- \\
-1-\end{array}$ & $\begin{array}{l}+1+ \\
-1- \\
-1- \\
-1- \\
-1-\end{array}$ & $\begin{array}{l}+/+ \\
-/- \\
-1- \\
-1- \\
-1-\end{array}$ & $\begin{array}{l}+/+ \\
+/+ \\
-/- \\
-/- \\
-/-\end{array}$ & $\begin{array}{l}+/+ \\
+/+ \\
-/- \\
+/+ \\
+/+\end{array}$ \\
\hline
\end{tabular}

Detection of gene product by PCR is denoted with a plus or minus for each animal. Thus, " $+/{ }^{\prime}$ " denotes detection of Sry in the first animal, but not the second. There was a rapid repopulation of the spleen (at 8 weeks) and, to a lesser extent, lung (at 30 weeks) in $Y \rightarrow X$ bone marrow transplantation. This repopulation was absent in the brain parenchyma and retina until 52 weeks after transplantation.

Two rats were killed at each time point: $8,10,12,30$, and 52 weeks after bone marrow transplantation. The animals were perfused with normal saline to remove any intravascular circulating cells. The retinas from each animal were used independently, yielding two retinal samples from each animal. Retinal DNA was extracted (Easy-DNA kit; Invitrogen, Carlsbad, CA, USA), and the pellet was dissolved in $50 \mu \mathrm{l}$ TRIS EDTA and quantified by $260 \mathrm{~nm}$ absorption. Exactly $1 \mu \mathrm{g}$ DNA was used for polymerase chain reaction (PCR). Similarly, DNA samples from the spleen, lungs, and brain were obtained and quantified.

As previously decribed, ${ }^{6}$ PCR amplification for 35 cycles of a 104 bp segment of the murine Sry gene (the sex determining region of the $\mathrm{Y}$ chromosome) encoded by genomic DNA isolated from harvested organs was performed using the following pair of primers: 5'-CATCGAAGGGTTAAAGTGCCA$3^{\prime}$ (5' primer) and 5'-ATAGTGTGTAGGTTGTTGTCC-3' (3' primer). A previously published experiment established the sensitivity of this method for detecting the presence of $104 \mathrm{bp}$ fragments of male DNA: this PCR protocol detected l ng of male DNA in $1 \mu \mathrm{g}$ of female DNA $(0.1 \%)$ and could detect $0.01 \%$ male cells $(1: 10000)$ in the mixture of male and female cells. ${ }^{6}$

\section{RESULTS}

Confirming the establishment of chimerism, PCR showed the presence of $\mathrm{Y}$ chromosome murine Sry gene product in spleens harvested from chimeric $(\mathrm{Y} \rightarrow \mathrm{X})$ Lewis rats 8 weeks after bone marrow transplantation, as well as at every time point thereafter (table 1). Sry was not detected in the lungs of chimeric rats 8,10 , or 12 weeks after establishment of chimerism; but Sry was detected in the lungs of chimeric rats at 30 weeks and 52 weeks. Sry could not be detected in retinas and brains of chimeric rats $8,10,12$, or 30 weeks after bone marrow transplantation. However, the retina of one eye from each animal did show the presence of Sry at 52 weeks, as did the brain (fig 1)

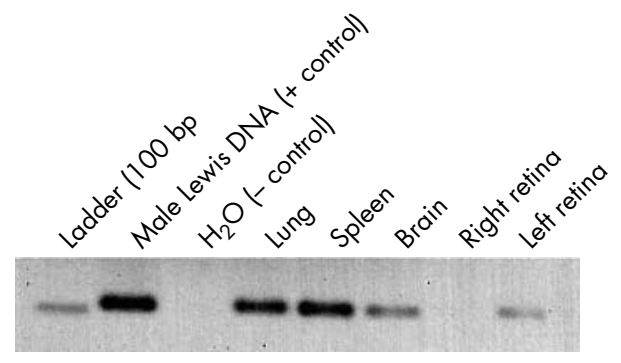

Figure 1 PCR amplification for 35 cycles of a $104 \mathrm{bp}$ segment of the murine Sry gene (the sex determining region of the $Y$ chromosome) from DNA extracted from organs of a bone marrow chimeric $(Y \rightarrow X)$ Lewis rat 52 weeks after bone marrow transplant. The 104 bp product is detected in the lung, spleen, brain, and left retina.

\section{DISCUSSION}

The origin of microglia in the CNS has been a focus of debate. However, the current prevailing view is that macrophage-like cells, presumably derived from mesodermal cells belonging to haematopoietic lineage, invade the brain parenchyma during embryonic development. ${ }^{12-14}$ Similarly, retinal microglia are the only bone marrow derived cells in the healthy retina when there is no infiltration or extravasation of bloodborne cells. Bone marrow chimeric $(\mathrm{Y} \rightarrow \mathrm{X})$ animals make it possible to use PCR for Y chromosome gene product to detect bone marrow derived cells in the retina with great sensitivity. This study shows that in the absence of inflammation, the retina and brain in bone marrow chimeric rats remains free of bone marrow derived cells for up to one year. This fact could be exploited to better characterise infiltrating cells in inflammatory reactions such as EAU.

PCR is a highly sensitive technique with some limitations. We have demonstrated that PCR allows for detection of $\mathrm{Y}$ chromosome gene product of a single XY cell in $10000 \mathrm{XX}$ cells. ${ }^{6}$ Assuming that $2 \%$ of retinal cells are microglia, we should be able to detect $Y$ chromosome gene product when as few as $0.5 \%$ of the microglia in the retina carry $\mathrm{Y}$ chromosomes. Not all positive amplification for $\mathrm{Y}$ gene product represents microglia; the presence of passenger leucocytes cannot be absolutely excluded despite saline perfusion of the animals before harvest of organs. Retinal stem cells or other mesenchymal cells are known migrate into the retina during embryonic development and may migrate into the adult uninflamed retina, although to the best of our knowledge adult migration has not been shown. Also, we cannot conclude that absolutely no bloodborne cells are present in retina and brain before 52 weeks; we may miss fewer than one bloodborne cell in 10000 cells. Nevertheless, we conclude that for at least 30 weeks after the establishment of chimerism, less than $0.5 \%$ of microglia are newly derived from the bone marrow. Interestingly, the retina of only one eye from each animal showed the presence of Sry at 52 weeks (fig 1) Since unilateral detection of Sry was seen in two animals and confirmed for both animals on two repeat runs of PCR, we do not think this represents a false positive. Alternatively, this may reflect numbers of infiltrating bloodborne cells in the retina at the threshold of yielding positive PCR.

Bone marrow chimera models are particularly useful for distinguishing resident microglia from newly infiltrating macrophages in retinal inflammation since there are no generally accepted helpful immunohistochemical markers to make this distinction. PCR technology and in situ hybridisation may make it possible to quantify and localise infiltrating cells; however this study sought to describe the relative rate of migration of bloodborne cells into the uninflammed retina, and therefore only the most sensitive technique was usedthat is, PCR. Confirmatory in situ hybridisation was likely to yield a false negative result because of the probable low 
number of bloodborne cells present in any given tissue section.

Finally, this study confirms the similarity in stability of microglial populations in the brain and retina. In previous studies using bone marrow chimeric rats to characterise CNS microglial turnover, parenchymal microglia were found to be stable 1 year after bone marrow transplantation. In these studies, meningeal monocytes, and to a lesser extent perivascular CNS microglia, were replaced by bone marrow derived cells more quickly. ${ }^{9}$ This was quantified in a study by Kennedy and Abkowitz ${ }^{15}$ : 1 month after transplantation, 89\% of the donor cells were detected in the spleen; after l year, $61 \%$ were detected in lung and $30 \%$ in perivascular and leptomeningeal sites, but not in the parenchyma of the brain. The retinal microglia were not studied. Since there are fewer parenchymal microglia in the retina than in the brain, it was unclear how quickly the predominantly perivascular retinal microglia would be replaced by bone marrow derived monocytes. This study shows that retinal microglia do not turn over quickly and suggests that, like CNS microglia, retinal microglia remain inactivated behind the blood-retina barrier for long periods of time and are uniquely adapted, in contradistinction to other tissue resident macrophages. This study also confirms that as in the CNS, bone marrow derived cells do not typically cross the blood-retina barrier in the healthy retina.

\section{Authors' affiliations}

T A Albini, R C Wang, B Reiser, E Zamir, G S Wu, N A Rao, Doheny Eye Institute, Keck School of Medicine, University of Southern California, Los Angeles, CA, USA

Supported in part by National Eye Institute Grant EY013253 and core grant 3040 from the National Institutes of Health, Bethesda, Maryland, and unrestistricted grant from Research to Prevent Blindness, New York, NY, USA.
Correspondence to: Narsing A Rao, Doheny Eye Institute, DVRC 211, 1450 San Pablo Street, Los Angeles, CA 90033, USA; nrao@usc.edu

Accepted for publication 14 December 2004

\section{REFERENCES}

1 Barger SW, Harmon AD. Microglial activation by Alzheimer amyloid precursor protein and modulation by apolipoprotein E. Nature 1997;388:878-81.

2 McGeer PL, Itagaki S, Boyes BE, et al. Reactive microglia are positive for HLADR in the substantia nigra of Parkinson's and Alzheimer's disease brains. Neurology 1988;38:1285-91.

3 Price RW, Brew B, Sidtis J, et al. The brain in AIDS: central nervous system HIV-1 infection and AIDS dementia complex. Science 1988;239:586-92.

4 Sriram S, Rodriguez M. Indictment of the microglia as the villain in multiple sclerosis. Neurology 1997;48:464-70.

5 Matsubara T, Pararajasegaram G, Wu GS, et al. Retinal microglia differentially express phenotypic markers of antigen-presenting cells in vitro. Invest Ophthalmol Vis Sci 1999:40:3186-93.

6 Rao NA, Kimoto T, Zamir E, et al. Pathogenic role of retinal microglia in experimental uveoretinitis. Invest Ophthalmol Vis Sci 2003;44:22-31.

7 Barron KD. The microglial cell. A historical review. J Neurol Sci 1995; 134(Suppl):57-68

8 Chen L, Yang P, Kiilstra A. Distribution, markers, and functions of retinal microglia. Ocul Immunol Inflamm 2002;10:27-39.

9 Lassmann H, Hickey WF. Radiation bone marrow chimeras as a tool to study microglia turnover in normal brain and inflammation. Clin Neuropathol 1993; 12:284-5.

10 Lassmann H, Schmied M, Vass K, et al. Bone marrow derived elements and resident microglia in brain inflammation. Glia 1993;7:19-24.

11 Flugel A, Bradl M, Kreutzberg GW, et al. Transformation of donor-derived bone marrow precursors into host microglia during autoimmune CNS inflammation and during the retrograde response to axotomy. J Neurosci Res 2001;66:74-82.

12 Eglitis MA, Mezey E. Hematopoietic cells differentiate into both microglia and macroglia in the brains of adult mice. Proc Natl Acad Sci USA 1997;94:4080-5

13 Ling EA, Wong WC. The origin and nature of ramied and amoeboid microglia: a historical review and current concepts. Glia 1993;7:9-18.

14 Santambrogio L, Belyanskaya SL, Fischer FR, et al. Developmental plasticity of CNS microglia. Proc Natl Acad Sci USA, 2001;98:6295-300.

15 Kennedy DW, Abkowitz JL. Kinetics of central nervous system microglial and macrophage engraftment: analysis using a transgenic bone marrow transplantation model. Blood 1997;90:986-93. 